\title{
Diagnostic accuracy of ultrasonography in comparison with colonoscopy for diagnosing of ulcerative colitis
}

\begin{abstract}
Aim: To evaluate diagnostic accuracy of ultrasonography in comparison with colonoscopy as gold standard for diagnosing of ulcerative colitis (UC).

Method: In this prospective study, 114 participants who attended in Ahvaz Imam Hospital during March 2014 to February 2015 included. The participants divided into 2 groups: group A as $64 \mathrm{UC}$ patients (flare or new case) and 50 participants as control group who attend for colorectal cancer screening or evaluation of rectorrhagia. All of the participants obtained a Trans abdominal ultrasonography after 8hours fasting and evaluated for colonic thickness in any involved region, loss of compressibility, peristaltic movement, mesenteric lymph nodes enlargement and presence or absence of ascites, fat stranding or stratification.

Results: Ultrasonography sensitivity for diagnosing of moderate to severe UC in comparison with colonoscopy was between 52.56 to $86.36 \%$ based on different factors such as new onset or active disease, BMI, gender and region of involvement. In all of the situations the specificity was $100 \%$. The sensitivity for diagnosing new cases of UC and pancolitis were $86.36 \%$ and $76.67 \%$ respectively and these figures were less among cases with BMI more than 25 . The colonic wall thickness in new cases of UC $(6.4 \pm 1.3 \mathrm{~mm})$ was significantly more than wall thickness among disease flare up $(5.5 \pm 1.7 \mathrm{~mm})(\mathrm{P}=0.02)$ and also average colon thickness in UC group (average $5.9 \mathrm{~mm}$, range $2-9 \mathrm{~mm}$ ) was significantly more than control group (average $3.3 \mathrm{~mm}$, range $3-4 \mathrm{~mm}$ ).
\end{abstract}

Conclusion: The findings of current study prove that ultrasonography is not an ideal tool for evaluation of UC patients because its results could affect by several factors such as BMI and region of involvement.

Keywords: ultrasonography, ulcerative colitis, colonoscopy, sensitivity, specificity
Volume 9 Issue 5 - 2018

\author{
Eskandar Hajiani,' 'Mohammad Momen \\ Gharibvand, ${ }^{2}$ Pezhman Alavinejad,' Elena \\ Lak, $^{3}$ Masoumeh Khedri ${ }^{3}$ \\ 'Research Center for Infectious Diseases of Digestive System, \\ Ahvaz Jundishapur University of Medical Sciences, Iran \\ ${ }^{2}$ Assistant professor of Radiology, Department of radiology, \\ Ahvaz Jundishapur University of Medical Sciences, Iran \\ ${ }^{3}$ Ahvaz Imam Hospital, Ahvaz Jundishapur University of Medical \\ Sciences, Iran
}

Correspondence: Dr Pezhman Alavinejad, Assistant professor of Gastroenterology and hepatology,

Ahvaz Jundishapur University of Medical Sciences, Ahvaz, Iran, Tel +98916 III 5880, Fax + 986132921839 ,

Email pezhmanalavinejad@gmail.com

Received: April 23, 2018 | Published: October 02, 2018

\section{Introduction}

Ulcerative colitis (UC) is a chronic inflammatory bowel disorder with characteristic courses of flare up and remission. ${ }^{1,2}$ The diagnosis of US is based on combination of clinical, colonoscopy, laboratory and imaging findings. Colonoscopy is the gold standard for diagnosis of this disorder but it is an invasive procedure and the risk of its complications such as perforation rising during acute presentation of disease. ${ }^{2-7}$ This issue emphasizes the importance of a noninvasive and accurate diagnostic method in clinical practice. ${ }^{3,8,9}$ None of the imaging modalities alone could qualify as gold standard for evaluation of all of the aspects of UC. . $^{9-11}$

Ultrasonography in comparison with endoscopy is noninvasive, inexpensive, radiation free, easy to use, acceptable, repeatable and completely compatible with an ideal diagnostic method. ${ }^{12}$ Tran abdominal ultrasonography is useful for diagnosing of bowel wall thickness, the extent of inflammatory involvement and assessment of disease activity. ${ }^{13,14}$ Diagnostic accuracy of ultrasonography in inflammatory bowel disease is highly depended on site of involvement and range from more than $90 \%$ in sigmoid and descending colon to very low rates in rectum. ${ }^{15}$

In literature, most of the studies have evaluated the role of the ultrasonography in Crohn's disease and the diagnostic efficacy of this method for ulcerative colitis has not evaluated thoroughly. ${ }^{12,16}$ In the current study, we evaluate the efficacy and accuracy of Tran abdominal ultrasonography in comparison with colonoscopy in diagnosing of ulcerative colitis.

\section{Method}

In this prospective study, the sensitivity and specificity of Tran abdominal ultrasonography in comparison with colonoscopy for diagnosing ulcerative colitis determined among 114 persons who attended in Ahvaz Imam Hospital as a referral center during March 2014 to February 2015. The participants divided into 2 groups as group A as ulcerative colitis patients (64 cases) and group B including the participants who attended for colonoscopy due to colorectal cancer screening or evaluation of rectorrhagia caused by any other reason than UC (50 cases). The demographic characters of participants recorded and after exclusion of intestinal infections or clostridium difficult in case of resent antibiotic usage, they sent for colonoscopy. All of the colonoscopies performed by an experienced gastroenterologist and the colon evaluated for any evidence of UC including hyperemia, granularity, loss of vascularity, erythematic, friability, erosions or oxidative ulceration and pseudo polyp formation.

After completion of colonoscopy examination and full colonoscopy, the participants referred for Tran abdominal gray scale 
ultrasonography by a radiologist (GE S6, probe $7-10 \mathrm{MHz}$ ) after 8hours fasting. During ultrasonography the thickness of descending colon or any other involved region determined and also they assessed for peristaltic movement activity including increase or decrease, loss of compressibility, mesenteric lymph node enlargement, absence or presence of ascites, fat stranding and or stratification. The maximum thickness of colon wall in ultrasonography considered as a marker of disease activity and pathologic process with cut off level of more than $4 \mathrm{~mm}$. loss of colonic haustration also considered abnormal. Biochemical markers of disease activity measured including ESR, CRP, Hb, Plt, WBC and stool Calprotectin level.

The colon involvement defined as proctosigmoiditis, left side colitis, extensive colitis and pancolitis. The radiologist was unaware of participants clinical and biochemical profiles and also detail of colonoscopy results. The time lag between colonoscopy and ultrasonography was less than 3 days. Exclusion criteria include abdominal ascites, history of GI malignancy, severe heart failure, pregnancy, BMI $>35$, any history of GI tract surgery and diagnosis of Crohn's disease. The results of ultrasonography compared with colonoscopy findings and the sensitivity and specificity determined by RUC curve model. The statistical analysis of DATA performed by SPSS software (version 22) and two tailed T-test with (significant $<0.05$ ).

\section{Results}

The demographic characters of the participants have been shown in Table 1. Based on sonographic findings (Table 2), colonic mural thickness among new cases of ulcerative colitis $(6.4 \pm 1.3 \mathrm{~mm})$ was significantly more than cases who diagnosed as UC flare up $(5.5 \pm 1.7 \mathrm{~mm})(\mathrm{P}=0.02)$ (Figure 1). The average mural thickness among subjects with active or new onset UC $(5.9 \mathrm{~mm}$, range $2-9 \mathrm{~mm})$ was significantly more than average value in control group $(3.3 \mathrm{~mm}$, range $3-4 \mathrm{~mm})(\mathrm{P}<0.0001)$ (Figure 2 ) (Figure 3 ). Figure 4 clarify sensitivity and specificity of Tran abdominal ultrasonography for diagnosing ulcerative colitis based on different variables such as sex, age, BMI, activity of disease and site of involvement. While the specificity was almost $100 \%$ for all of the variables, the sensitivity in descending order was related to BMI $<25$, new onset course of disease, male gender and pancolitis.

In univariable logistic regression model, there was a statistically significant relation between mural thickness and results of ultrasonography $(\mathrm{P}<0.0001)$. In multivariate logistic regression model, this meaningful relation found between sonographic report and variables such as $\mathrm{BMI}$ and peristaltic movement $(\mathrm{P}=0.047$ \& 0.002 respectively) while other variables such as sex, age and disease activity had not any significant relation $(\mathrm{P}>0.05)$.

Table I The Demographic characteristics of 2 groups

\begin{tabular}{llll}
\hline Variable & Group A (UC patients) $(\mathbf{n}=\mathbf{6 4})$ & Group B (Screening colonoscopy) $(\mathbf{n}=\mathbf{5 0})$ & P Value \\
\hline Gender $(\mathrm{m} / \mathrm{f})$ & $40 / 24$ & $31 / 19$ & 0.9 \\
$\mathrm{Age}(\mathrm{y})$ & $35.13 \pm 11.0$ & $54.09 \pm 4.8$ & 0.001 \\
$\mathrm{BMI}\left(\mathrm{kg} / \mathrm{m}^{2}\right)$ & $24.08 \pm 2.9$ & $21.5 \pm 2.1$ & 0.001
\end{tabular}

Table 2 Sonographic findings among patients diagnosed with colitis ulcerative (group A) and control group (B); UC, ulcerative colitis, NA, nonaccessible, mm, millimeter

\begin{tabular}{lllllll}
\hline \multirow{2}{*}{ Characteristics } & \multicolumn{2}{c}{ Group A (UC) } & \multicolumn{2}{c}{ Group B (Control) } & \\
\cline { 2 - 5 } & $\mathbf{N}$ & $\%$ & $\mathbf{N}$ & $\%$ & P Value \\
\hline Stratification & 39 & 39.1 & 0 & 0 & NA \\
Prestalic motion & 42 & 65.6 & 0 & 0 & NA \\
Mesenteric lymphadenopathy & 10 & 15.6 & 0 & 0 & NA \\
Fat stranding & 30 & 46.9 & 0 & 0 & NA \\
No compression & 34 & 46.9 & 0 & 0 & NA \\
Intestine thickness (mm) & $5.9 \pm 1.6$ & $2-9$ & $3.3 \pm 0.4$ & $3-4$ & $<0.000$ I \\
New case & $6.4 \pm 1.3$ & & - & - & 0.02 \\
Flare up & $5.5 \pm 1.7$ & & & & \\
\hline
\end{tabular}




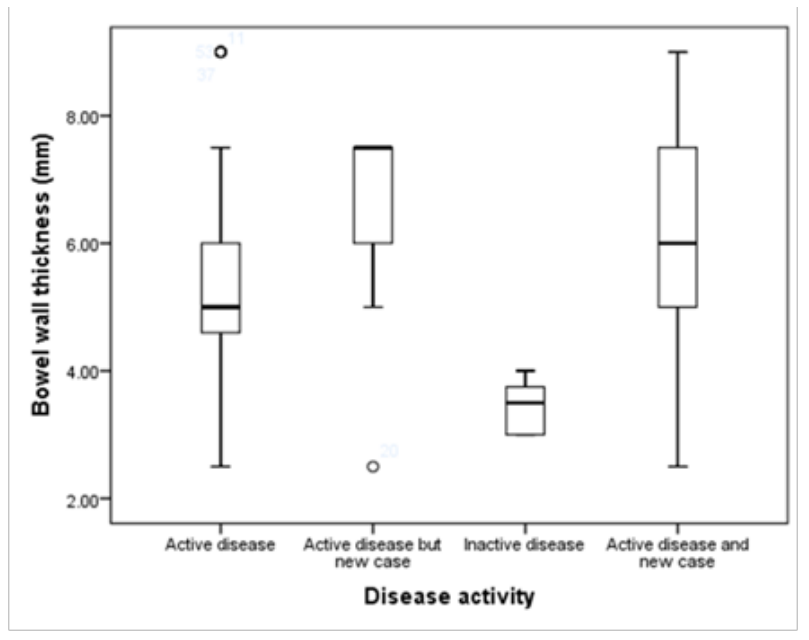

Figure I Relation between disease activity and sonographic records of bowel wall thickness.

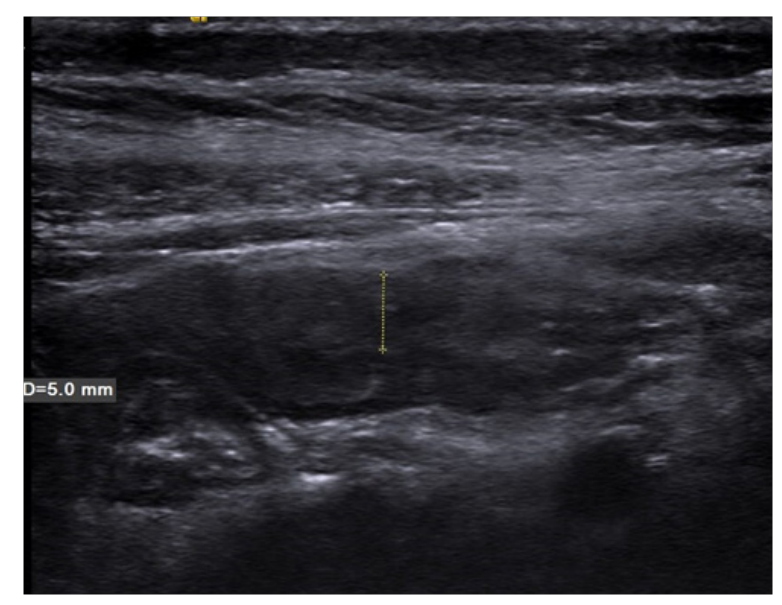

Figure 2 Sonographic appearance of inflamed colon segment in ulcerative colitis. Findings include thickened bowel wall, loss of stratification and narrow lumen.

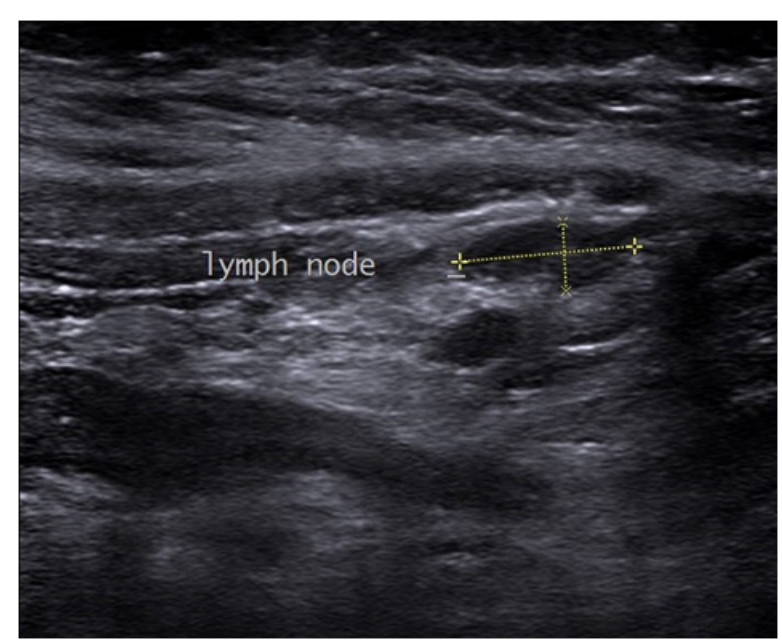

Figure 3 Thickening of sigmoid wall with enlarged mesenteric lymph node.

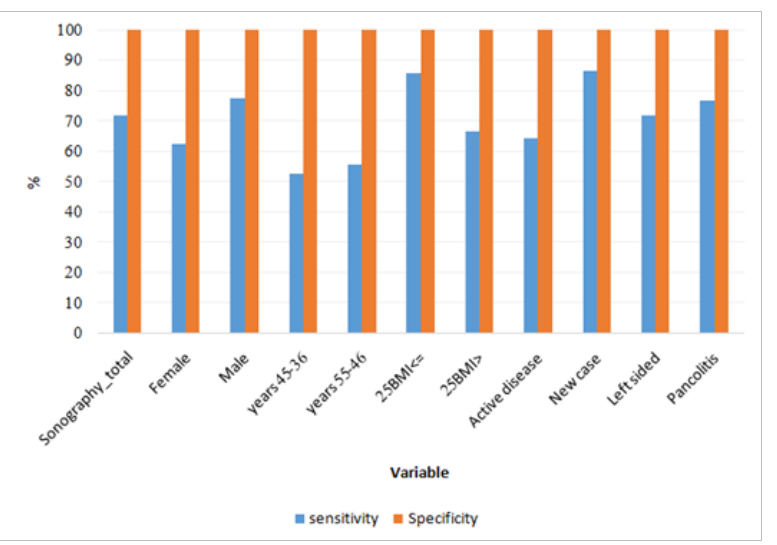

Figure 4 Sensitivity and specificity of Transabdominal ultrasonography for diagnosing ulcerative colitis based on different variables.

\section{Discussion}

Managing ulcerative colitis necessitate objective methods not only during control of active disease but also during follow up for better detection of disease activity and determining the site of involvement. Currently colonoscopy is the standard method for evaluating ulcerative colitis activity, severity and expansion but this procedure is invasive and during sever disease flare could be non-applicable due to fear of complications such as bleeding and perforation. ${ }^{6,7}$ Moreover many patients are reluctant to endoscopic procedures during each flare. So, availability of an accurate noninvasive method for determining disease activity and extension is mandatory.

In literature, the ultrasonography features of ulcerative colitis have been reported to be alteration of bowel wall echo pattern, loss of haustra coliand specially bowel wall thickening of more than $4 \mathrm{~mm}$ which has been strongly correlated with C-reactive protein values and the endoscopic score. ${ }^{17,18}$ It is supposed that Tran abdominal ultrasonography could be applicable for determining active bowel inflammation among UC patients. ${ }^{10-16}$ Although specificity of the ultrasonography in differentiating UC from other reasons of bowel inflammation is low and itssensitivity has been reported to be more than $90 \% .^{15,19}$ The range of sensitivity and specificity have been between 48 to $100 \%$ and 82 to $90 \%$ respectively with highest reported sensitivity in sigmoid and descending colon (up to 97\%) and the least in rectum (almost 15\%). ${ }^{14,15,20}$ Prove of high accordance rate between endoscopic disease activity index and ultrasonography score could show that ultrasonography is capable for determining of mucosal healing among ulcerative colitis. ${ }^{14,21,22}$

The clinical and ultrasonography findings of early stages are not unique toulcerative colitis and could be found in other inflammatory disorders such as drug induced colitis, infectious colitis, Crohn's disease or ischemic colitis. So the current study designed to evaluate just ulcerative colitis. Recently a review article suggested 3 indications for utility of ultrasonography in ulcerative colitis: assessing the extent of the disease in cases of incomplete colonoscopy, aid in the differential diagnosis in cases of indeterminate colitis in order to rule out small intestine involvement and eventually as an alternative to colonoscopy inoutbreaks of the disease. ${ }^{23}$ In the current study, the sensitivity of ultrasonography for diagnosing moderate to severe 
colitis was between 52.56 to $86.36 \%$ based on different factors such as new onset or flare of disease, BMI, gender and site of involvement. The specificity in all of the situations was $100 \%$. The sensitivity for diagnosing new cases of UC was $86.36 \%$ and with BMI less than 25 , male gender and pancolitis was $85.72 \%, 77.5 \%$ and $76.67 \%$ respectively which is in concordance with other studies. Although the reported sensitivity is less than results of some other studies which could be related to the low resolution of ultrasonography unit.

Contrast enhanced ultrasonography has a high sensitivity for diagnosing colitis but was unsuccessful in getting popularity dueto technical difficulties. For evaluation of disease activity by ultrasonography, the best investigated variables are mural thickness and blood flow in colon wall but ultrasonography fails to diagnose superficial abnormalities like mucosal erythema and decrease in vascular pattern. Moreover the colon wall thickness significantly increases from right to left $(\mathrm{P}=0.001)$ which could be a logic explain of ultrasonography sensitivity difference $(75 \%)$ and left side colon $(96 \%)$.

In the current study, the ultrasonography disease activity determined based on variables such as mural thickness, absence of haustration, presence of mesenteric lymphadenopathy, peristaltic motion, fat stranding and loss of compressibility. Some of the studies have shown a significant difference between colon wall thickness among cases with moderate to severe colitis and cases of mild or inactive colitis either before or after treatment. ${ }^{24,25} \mathrm{In}$ our study the mural thickness of colon among new cases of UC $(6.4 \pm 1.3 \mathrm{~mm})$ was significantly more than this value in cases who diagnosed as UC flare $(5.5 \pm 1.7 \mathrm{~mm}$, $\mathrm{P}=0.02$ ). Moreover average thickness of colon wall in active or new cases of UC (average 5.9 , range $2-9 \mathrm{~mm}$ ) was significantly more than mural thickness in control group (average 3.3, range 3-4mm).

The study of Parento et al. ${ }^{14}$ on 83 adult patients with moderate to severe UC reported that colon thickness is in concordance with disease activity. ${ }^{14}$ Civitelli et al., ${ }^{12}$ found a meaningful difference of colon wall thickness between normal parts and segments with moderate to severe inflammation ${ }^{12}$ although in case of mild inflammation, the difference is not significant. These results are in concordance with the findings of current study.

This study in contrast to most of the previous studies was prospective and cross sectional and the patients were homogenous group as moderate to severe colitis. One of the limitations of this study was performing ultrasonography by just one radiologist. The other one was unicenteric location of the study, while a multicentric study with participation of 2 or more sonographists could further potentiate results of this assessment. Based on differences of ultrasonography sensitivity according to patients BMI and type of the disease as pancolitis or left side colitis, it is clear that ultrasonography could not be recommended as an ideal method for evaluation of ulcerative colitis.

\section{Conclusion}

Findings of current study reveal that ultrasonography is not an ideal method for evaluation of UC patients and the impact of BMI and location of the disease on the sensitivity of ultrasonography was obvious.

\section{Acknowledgements}

None.

\section{Conflict of interest}

The author declares no conflict of interest.

\section{References}

1. Andres PG, Friedman LS. Epidemiology and the natural course of inflammatory bowel disease. Gastroenterology Clinics of North America. 1999;28(2):255-281

2. Kornbluth A, Sachar DB. Ulcerative colitis practice guidelines in adults: American college of gastroenterology, practice parameters committee. Am J Gastroenterol. 2010;105(3):501-523.

3. Horsthuis K, Bipat S, Bennink RJ, et al. Inflammatory bowel disease diagnosed with US, MR, scintigraphy, and CT: meta-analysis of prospective studies 1. Radiology. 2008;247(1):64-79.

4. Wu L, Li P, Wu J, et al. The diagnostic accuracy of chromoendoscopy for dysplasia in ulcerative colitis: meta $\square$ analysis of six randomized controlled trials. Colorectal Dis. 2012;14(4):416-420.

5. Bernstein CN, Fried M, Krabshuis JH, et al. World gastroenterology organization practice guidelines for the diagnosis and management of IBD in 2010. Inflamm Bowel Dis. 2010;16(1):112-124.

6. Navaneethan U, Kochhar G, Phull H, et al. Severe disease on endoscopy and steroid use increase the risk for bowel perforation during colonoscopy in inflammatory bowel disease patients. J Crohns Colitis. 2012;6(4):470-475.

7. Navaneethan U, Parasa S, Venkatesh PG, et al. Prevalence and risk factors for colonic perforation during colonoscopy in hospitalized inflammatory bowel disease patients. J Crohns Colitis. 2011;5(3):189-195.

8. Conti CB, Giunta M, Gridavilla D, et al. Role of bowel ultrasound in the diagnosis and follow-up of patients with Crohn's Disease. Ultrasound in Medicine \& Biology. 2017;43(4):725-734.

9. Haas K, Rubesova E, Bass D. Role of imaging in the evaluation of inflammatory bowel disease: How much is too much? World J Radiol. 2016;8(2):124

10. Strobel D, Goertz RS, Bernatik T. Diagnostics in inflammatory bowe disease: ultrasound. World J Gastroenterol. 2011;17(27):3192-3197.

11. Nylund K, Hausken T, Gilja OH. Ultrasound and inflammatory bowel disease. Ultrasound quarterly. 2010;26(1):3-15.

12. Civitelli F, Di Nardo G, Oliva S, et al. Ultrasonography of the colon in pediatric ulcerative colitis: a prospective, blind, comparative study with colonoscopy. J Pediatr. 2014;165(1):78-84.

13. Di Sabatino A, Armellini E, Corazza GR. Doppler sonography in the diagnosis of inflammatory bowel disease. Dig Dis. 2004;22(1):63-66.

14. Parente F, Molteni M, Marino B, et al. Are colonoscopy and bowel ultrasound useful for assessing response to short-term therapy and predicting disease outcome of moderate-to-severe forms of ulcerative colitis?: a prospective study. Am J Gastroenterol. 2010;105(5):1150 1157.

15. Parente F, Greco S, Molteni M, et al. Role of early ultrasound in detecting inflammatory intestinal disorders and identifying their anatomical location within the bowel. Aliment Pharmacol Ther. 2003;18(10):10091016.

16. Dietrich CF. Significance of abdominal ultrasound in inflammatory bowel disease. Dig Dis. 2009;27(4):482-493.

17. Bezzio C, Furfaro F, Monteleone M, et al. Intestinal ultrasound in ulcerative colitis. In Imaging of Ulcerative Colitis. Springer Milan; 2014. p. 15-21. 
18. Antonelli E, Giuliano V, Casella G, et al. Ultrasonographic assessment of colonic wall in moderate-severe ulcerative colitis: comparison with endoscopic findings. Digestive and Liver Disease. 2011;43(9):703-706.

19. Ceriotti S, Zucca E, Stancari G, et al. Sensitivity and specificity of ultrasonographic evaluation of small intestine wall thickness in the diagnosis of inflammatory bowel disease in horses: a retrospective study. Journal of Equine Veterinary Science. 2016;37:6-10.

20. Ordás I, Rimola J, García-Bosch O, et al. Diagnostic accuracy of magnetic resonance colonography for the evaluation of disease activity and severity in ulcerative colitis: a prospective study. Gut. 2013;62(11):1566-1572.

21. Parente F, Molteni M, Marino B, et al. Bowel ultrasound and mucosal healing in ulcerative colitis. Dig Dis. 2009;27(3):285-290.
22. Pascu M, Roznowski AB, Müller HP, et al. Clinical relevance of transabdominal ultrasonography and magnetic resonance imaging in patients with inflammatory bowel disease of the terminal ileum and large bowel. Inflamm Bowel Dis. 2004;10(4):373-382.

23. Poza-Cordón J, Ripollés-González T. Utility of abdominal ultrasonography in the diagnosis and monitoring of inflammatory bowel disease. Rev Esp Enferm Dig. 2014;106(6):395-408.

24. Maconi G. Ultrasonography in the evaluation of extension, activity, and follow-up of ulcerative colitis. Scand J Gastroenterol. 1999;34(11):1103-7.

25. Deepak P, Bruining DH. Radiographical evaluation of ulcerative colitis. Gastroenterology report. 2014;2(3):169-177. 